\title{
Approximations by Graphs and Emergence of Global Structures
}

\author{
P. EXNER ${ }^{a, b}$, P. HeJČÍK ${ }^{c}$ AND P. ŠEBA ${ }^{b, c}$ \\ ${ }^{a}$ Nuclear Physics Institute, Czech Academy of Sciences \\ 25068 Rež near Prague, Czech Republic \\ ${ }^{b}$ Doppler Institute, Czech Technical University \\ Břehová 7, 11519 Prague, Czech Republic \\ ${ }^{c}$ University of Hradec Králové, Víta Nejedého 573 \\ 50002 Hradec Králové, Czech Republic
}

\begin{abstract}
We study approximations of billiard systems by lattice graphs. It is demonstrated that under natural assumptions the graph wave functions approximate solutions of the Schrödinger equation with energy rescaled by the billiard dimension. As an example, we analyze a Sinai billiard with attached leads. The results illustrate emergence of global structures in large quantum graphs and offer interesting comparisons with patterns observed in complex networks of a different nature.
\end{abstract}

PACS numbers: 03.65.Nk

\section{Introduction}

The notion of a quantum graph is known for more than half a century [1], however, an intense investigation of these structures started less than two decades ago $[2,3]$ as a response to progress in fabrication technologies which allowed one to prepare microscopic graph-like structures. Nowadays, there is an extensive literature devoted to the subject; for recent reviews see $[4,5]$ and also [6].

An attention to quantum graphs comes from the fact that motion on their edges is easy to describe, and at the same time the graph structure leads to a nontrivial behavior. It was shown, in particular, that even a graph with a small number of vertices is capable of developing an internal dynamics rich enough to display universality features that are typical of the wave-chaotic behavior [7-9]. It is not only a theory, the results can be checked experimentally in a microwave graph model [10].

On the contrary, properties of nontrivial large-scale graphs have been regarded as less interesting due to the expected localization of the corresponding wave functions. An indication that this belief is wrong may be seen from the 
fact that complex graph-like structures, such as systems of interconnected neurons, display surprising patterns observed, for instance, in the visual cortex of mammals [11]. It was shown in [12] that these patterns can be understood as a manifestation of a Gaussian random field, and are in this sense analogous to patterns emerging in two-dimensional quantum chaotic systems, for instance, nodal domains in a chaotic quantum billiard [13].

A thorough investigation of such structures on graphs is by no means easy. To follow the mentioned example, a nodal domain is a connected component of the maximal induced subgraph of a graph $\Gamma$ on which a function does not change a sign; it relates the pattern formation on $\Gamma$ to nontrivial algebraic questions about graph partition, etc. This is probably the reason why only few mathematical results of this type are available at present, cf. [14, 15].

In this paper we are going to show that extended graphs support structures similar to those known from two-dimensional wave chaos and Gaussian randomfield models. Our approach is based on graph embedding into an Euclidean space and a convergence argument; we will demonstrate that wave functions on the graph can approximate solutions of the respective "continuous" billiard problem. The embedding assumption is naturally a nontrivial restriction because not every graph can be regarded as a subset of an Euclidean space from which it inherits its metric. It applies, however, to wide enough class of systems and allows us at the same time to circumvent difficulties of a pure algebraic treatment.

The technical tool to derive the approximation result is a graph duality adopted from [16]. To make the paper self-contained we review this theory in the next section in a simplified form suitable for the present purpose. Then we will show how solutions to the Schrödinger equation can be approximated by those of a Schrödinger equation on lattice graphs with the energy properly rescaled.

To illustrate the result we will analyze an example of a lattice graph which approximates a Sinai billiard. Since we want to go beyond the nodal structure and to analyze also the phase behavior of the wave functions we will study the graph also from the transport point of view, attaching to it a pair of semiinfinite leads; the result will be compared to the "true" Sinai billiard with a pair of leads attached. We will compare, in particular, the probability currents and show they are similar to each other provided the current on the graph is properly defined as a vector sum of currents at graphs links.

\section{Theory: a graph duality}

By $\Gamma$ we denote in the following a connected graph consisting of at most countable families of vertices $\mathcal{V}=\left\{\mathcal{X}_{j}: j \in I\right\}$ and edges $\mathcal{L}=\left\{\mathcal{L}_{j n}:(j, 0) \in\right.$ $\left.I_{\mathcal{L}} \subset I \times I\right\}$. We suppose that each pair of vertices is connected by not more than one link, otherwise we can simply add vertices to any "multiple" edge. The set $\mathcal{N}\left(\mathcal{X}_{j}\right)=\left\{\mathcal{X}_{n}: n \in \nu(j) \subset I \backslash\{j\}\right\}$ consists of the neighbors of $\mathcal{X}_{j}$, i.e. the vertices connected with $\mathcal{X}_{j}$ by a single edge is nonempty by assumption. The 
graph boundary $\mathcal{B}$ consists of vertices having a single neighbor; it may be empty. We denote by $I_{\mathcal{B}}$ and $I_{\mathcal{I}}$ the index subsets in $I$ corresponding to $\mathcal{B}$ and the graph interior $\mathcal{I}:=\mathcal{V} \backslash \mathcal{B}$, respectively.

We suppose that $\Gamma$ is a metric graph, i.e. that it has a local metric structure, every edge $\mathcal{L}_{j n}$ being isometric with a line segment $\left[0, \ell_{j n}\right]$. Of course, the graph can be also equipped with a global metric, for instance, by identifying it with a subset of $R^{\nu}$. In general the metrics may not coincide, however, in the next section we will identify them. Using the local metric, we introduce the Hilbert space $L^{2}(\Gamma):=\bigoplus_{(j, n) \in I_{\mathcal{L}}} L^{2}\left(0, \ell_{j n}\right)$ whose elements are $\psi=\left\{\psi_{j n}:(j, n) \in I_{\mathcal{L}}\right\}$ or simply $\left\{\psi_{j n}\right\}$; in the same way we define Sobolev spaces on $\Gamma$. Given a family of potentials $U:=\left\{U_{j n}\right\}$ with $V_{j n} \in L^{\infty}\left(0, \ell_{j n}\right)$ and coupling constants $\alpha:=\left\{\alpha_{j} \in R: j \in I\right\}$, we define the Schrödinger operator $H_{\alpha} \equiv H_{\alpha}(\Gamma, U)$ by

$$
H_{\alpha}\left\{\psi_{j n}\right\}:=\left\{-\psi_{j n}^{\prime \prime}+U_{j n} \psi_{j n}:(j, n) \in I_{\mathcal{L}}\right\}
$$

on the domain consisting of all $\psi$ with $\psi_{j n} \in W^{2,2}\left(0, \ell_{j n}\right)$ satisfying suitable boundary conditions at the vertices linking the boundary values

$$
\psi_{j n}(j):=\lim _{x \rightarrow 0+} \psi_{j n}(x), \quad \psi_{j n}^{\prime}(j):=\lim _{x \rightarrow 0+} \psi_{j n}^{\prime}(x),
$$

where the point $x=0$ is identified with $\mathcal{X}_{j}$. Specifically, we will work here with the so-called $\delta$ coupling: at any $\mathcal{X}_{j} \in \mathcal{V}$ we have $\psi_{j n}(j)=\psi_{j m}(j):=\psi_{j}$ for all $n, m \in \nu(j)$, and

$$
\sum_{n \in \nu(j)} \psi_{j n}^{\prime}(j)=\alpha_{j} \psi_{j}
$$

it is known that among all (nontrivial) boundary conditions which make the operator (2.1) self-adjoint there are no other with wave functions continuous at the vertices [3]. The particular case $\alpha=0$ represents the most simple boundary conditions, called usually Kirchhoff [4], which we will employ in the example of Sect. 4, however, for the moment it is useful to consider the more general situation (2.3). Furthermore, if the boundary $\mathcal{B} \neq \emptyset$ we assume Dirichlet boundary conditions there,

$$
\psi_{j}=0, \quad j \in I_{\mathcal{B}} .
$$

If $\Gamma$ is infinite one can look not only for bound states of $H_{\alpha}$ but also for solutions of the equation

$$
H_{\alpha} \psi=k^{2} \psi
$$

referring to the continuous spectrum. To describe the generalized eigenfunctions we consider in such a case the class $D_{\text {loc }}\left(H_{\alpha}\right)$ which is the subset in $\bigvee_{(j, n) \in I_{\mathcal{L}}} L^{2}\left(0, \ell_{j n}\right)$ (the direct sum) consisting of the functions which satisfy all the requirements imposed at $\psi \in D\left(H_{\alpha}\right)$ except the global square integrability. The conditions (2.3) define self-adjoint operators also if the $\alpha_{j}$ 's are formally put equal to infinity. We exclude this possibility, which corresponds to Dirichlet decoupling of the operator at $\mathcal{X}_{j}$ turning the vertex effectively into $N_{j}$ points of the boundary. 
We need the decoupling, however, to state the result. Let $H_{\alpha}^{D}$ be the operators obtained from $H_{\alpha}$ by changing the conditions (2.3) at the points of $\mathcal{I}$ to Dirichlet and denote $\mathcal{K}:=\left\{k: k^{2} \in \sigma\left(H_{\alpha}^{D}\right)\right\}$. In the particular case when the particle is free at graph edges, $U_{j n}=0$, this set is given explicitly as $\mathcal{K}:=\left\{\pi n \ell_{j n}^{-1}:(j, n) \in \mathcal{I}_{\mathcal{L}}, n \in N_{+}\right\}$. We will adopt several assumptions, namely

(i) all the potentials of the family $\left\{U_{j n}\right\}$ are uniformly bounded for $(j, n) \in \mathcal{I}_{\mathcal{L}}$,

(ii) $\ell_{0}:=\inf \left\{\ell_{j n}:(j, n) \in \mathcal{I}_{\mathcal{L}}\right\}>0$,

(iii) $L_{0}:=\sup \left\{\ell_{j n}:(j, n) \in \mathcal{I}_{\mathcal{L}}\right\}<\infty$,

(iv) $N_{0}:=\max \{\# \nu(j): j \in I\}<\infty$.

To formulate the result, we need a few more notions. On $\mathcal{L}_{n j} \equiv\left[0, \ell_{j n}\right]$, where the right end point identified with the vertex $\mathcal{X}_{j}$, we denote as $u_{j n}$ and $v_{j n}$ the solutions to $-f^{\prime \prime}+U_{j n} f=k^{2} f$ which satisfy the normalized Dirichlet boundary conditions

$$
u_{j n}\left(\ell_{j n}\right)=1-\left(u_{j n}\right)^{\prime}\left(\ell_{j n}\right)=0, \quad v_{j n}(0)=1-\left(v_{j n}\right)^{\prime}(0)=0 ;
$$

their Wronskian is naturally equal to $W_{j n}=-v_{j n}\left(\ell_{j n}\right)=u_{j n}(0)$. After this preliminary we can specify the result of [16] to the present situation.

Theorem : (a) Let assumptions $(i)-(i v)$ be satisfied and suppose that $\psi \in D_{\text {loc }}\left(H_{\alpha}\right)$ solves $(2.5)$ for some $k \notin \mathcal{K}_{\alpha}$ with $k^{2} \in R, \operatorname{Im} k \geq 0$. Then the corresponding boundary values (2.2) satisfy the equation

$$
\sum_{n \in \nu(j) \cap I_{\mathcal{I}}} \frac{\psi_{n}}{W_{j n}}-\left(\sum_{n \in \nu(j)} \frac{\left(v_{j n}\right)^{\prime}\left(\ell_{j n}\right)}{W_{j n}}-\alpha_{j}\right) \psi_{j}=0 .
$$

Conversely, any solution $\left\{\psi_{j}: j \in I_{\mathcal{I}}\right\}$ of the system (2.6) determines a solution of (2.5) by

$$
\begin{aligned}
& \psi_{j n}(x)=\frac{\psi_{n}}{W_{j n}} u_{j n}(x)-\frac{\psi_{j}}{W_{j n}} v_{j n}(x) \quad \text { if } n \in \nu(j) \cap I_{\mathcal{I}}, \\
& \psi_{j n}(x)=-\frac{\psi_{j}}{W_{j n}} v_{j n}(x) \text { if } n \in \nu(j) \cap I_{\mathcal{B}} .
\end{aligned}
$$

(b) Under $(i),(i i), \psi \in L^{2}(\Gamma)$ implies that the solution $\left\{\psi_{j}\right\}$ of the system (2.6) belongs to $\ell^{2}\left(I_{\mathcal{I}}\right)$.

(c) The opposite implication is valid provided (iii), (iv) also hold, and $k$ has a positive distance from $\mathcal{K}$.

\section{Approximation by lattice graphs}

As the next step, let us inspect how the above duality looks under simplifying assumption: we will suppose that (a) all the graph edges have the same length $\ell>0$ and (b) all the potentials $U_{j n}$ vanish. Then the "elementary" solutions can be made explicit

$$
u_{j n}(x)=\frac{1}{k} \sin k(x-\ell), \quad v_{j n}(x)=\frac{1}{k} \sin k x
$$


with the Wronskian $W_{j n}=-\frac{1}{k} \sin k \ell$, and the dual system of equations (2.6) becomes

$$
-\sum_{n \in \nu(j)} \frac{\psi_{n}-\psi_{j} \cos k \ell}{k^{-1} \sin k \ell}+\alpha_{j} \psi_{j}=0, \quad j \in I
$$

let us notice that this is true even if some of the $\nu(j)$ correspond to points of the boundary, because we assume Dirichlet condition (2.4) there, so the corresponding $\psi_{n}$ 's are zero.

So far we worked with the local metric on $\Gamma$, now we will regard the graph as a subset in $R^{\nu}$ and assume that the local metric coincides with the global one obtained by this embedding. We will not strive for a most general result and concentrate on an important particular case of a cubic lattice graph $\mathcal{C}^{\nu} \equiv \mathcal{C}^{\nu}(\ell) \subset$ $R^{\nu}$ whose vertices are points $\left\{x_{j}(\ell)=\left(j_{1} \ell, \ldots, j_{\nu} \ell\right): j_{i} \in Z\right\}$ while the edges are segments connecting pairs of vertices in which values of a single index $j_{i}$ differ by one, and its subgraphs.

Theorem : Let $V: R^{\nu} \rightarrow R$ be a smooth function with $\nabla V$ bounded. Put $\alpha_{j}(\ell):=V\left(x_{j}\right) \ell$ and consider the family of operators $H_{\alpha}\left(\mathcal{C}^{\nu}(\ell), 0\right)$ with $\ell>0$. Suppose that for any fixed $\ell$ and $k$ with $k^{2} \in R, \operatorname{Im} k \geq 0$, the family $\left\{\psi_{j}^{\ell}\right\}$ solves the equation (3.1), and defines a step function $\psi_{\ell}: R^{\nu} \rightarrow C$ by

$$
\psi_{\ell}(x):=\psi_{j}^{\ell} \quad \text { if } \quad-\frac{1}{2} \ell \leq\left(x-x_{j}\right)_{i}<\frac{1}{2} \ell .
$$

Suppose that the family $\left\{\psi_{\ell}\right\}$ converges to a function $\psi: R^{\nu} \rightarrow C$ as $\ell \rightarrow 0$ in the sense that the quantities $\varepsilon_{j}(\ell):=\psi\left(x_{j}\right)-\psi_{\ell}\left(x_{j}\right)$ behave as

$$
\sum_{n \in \nu(j)}\left(\varepsilon_{n}(\ell)-\varepsilon_{j}(\ell)\right)=o\left(\ell^{2}\right),
$$

then the limiting function solves the equation

$$
-\Delta \psi(x)+V(x) \psi(x)=\nu k^{2} \psi(x) .
$$

Proof : Let $f$ be a $C^{2}$-smooth function, using its Taylor expansion to the second order we find

$$
\frac{f(x+\ell)-f(x-\ell)-2 f(x) \cos k \ell}{\ell k^{-1} \sin k \ell}=\frac{2 k}{\ell} f(x) \tan \frac{k \ell}{2}+f^{\prime \prime}(x) \frac{k \ell}{\sin k \ell}+o(\ell),
$$

so the right-hand side tends to $f^{\prime \prime}(x)+k^{2} f(x)$ as $\ell \rightarrow 0$; in fact, the error is $\mathcal{O}\left(\ell^{2}\right)$ provided $f \in C^{3}$. Applying this result to the function $\psi$ with respect to each of the $\nu$ variables and combining it with the fact that the family $\left\{\psi_{\ell}\left(x_{j}\right)\right\}$ solves the equation (3.1) we find

$$
\begin{aligned}
\Delta \psi\left(x_{j}\right)+\nu k^{2} \psi\left(x_{j}\right)-V\left(x_{j}\right) \psi\left(x_{j}\right) \\
=\left(\frac{\ell}{k} \sin k \ell\right)^{-1} \sum_{n \in \nu(j)}\left(\varepsilon_{n}(\ell)-\varepsilon_{j}(\ell)\right)+o(\ell)
\end{aligned}
$$

and the right-hand side tends to zero by assumption. 
Let us add a few comments:

(a) The requirement $k \notin \mathcal{K}$ means no restriction here, because for a fixed $k$ it is satisfied if $\ell$ is small enough.

(b) Notice that the limiting energy has to be rescaled to $\nu k^{2}$, where $\nu$ is the dimension, roughly speaking because all "local" momentum components are equal. This does not mean that the particle cannot move through such lattice in any possible angle in a zig-zag way.

(c) To illustrate the last claim recall how Fermi surface looks like on a 2D square lattice in the free case, $\alpha_{j}=0$ for any $j \in I$. By [17] it is described by the equation

$$
\cos \theta_{1} \ell+\cos \theta_{2} \ell=2 \cos k \ell
$$

where $\theta_{i}$ are the quasimomentum components, thus for small $\ell$ we have at the bottom of the spectrum

$$
2 k^{2}=\theta_{1}^{2}+\theta_{2}^{2}+\mathcal{O}\left(\ell^{2}\right)
$$

which looks like the free "continuous" motion, apart of the factor 2 multiplying the energy.

A similar result can be derived if the lattice graphs do not cover the whole $R^{\nu}$. Consider an open set $\Omega \subset R^{\nu}$ and call $\mathcal{C}_{\Omega}^{\nu} \equiv \mathcal{C}_{\Omega}^{\nu}(\ell)$ the subgraph of $\mathcal{C}^{\nu}(\ell)$ whose vertices are all points $x_{j}$ contained in $\Omega$. Let $\mathcal{P}_{\Omega}^{\nu}(\ell)$ denote the union of all closed hypercubes of $\mathcal{C}_{\Omega}^{\nu}(\ell)$, i.e. the "volume" of such a lattice in $R^{\nu}$. If an edge of $\mathcal{C}_{\Omega}^{\nu}(\ell)$ belongs to the boundary of $\mathcal{P}_{\Omega}^{\nu}(\ell)$ we delete it. It may also happen that $\mathcal{P}_{\Omega}^{\nu}$ is non-convex, i.e. there is an axis along which a boundary point has neighbors in $\mathcal{C}_{\Omega}^{\nu}(\ell)$ in both directions, then we regard the corresponding vertex as a family of disconnected vertices belonging to the boundary of $\mathcal{C}_{\Omega}^{\nu}(\ell)$; we call the lattice modified in this way $\tilde{\mathcal{C}}_{\Omega}^{\nu}(\ell)$. Mimicking the above argument, we arrive at the following conclusion:

Theorem : Suppose that the potential $V: \Omega \rightarrow R$ is smooth with $\nabla V$ bounded and set $\alpha_{j}(\ell):=V\left(x_{j}\right) \ell$. Let us consider the dual system associated with the family $\left\{H_{\alpha}\left(\tilde{\mathcal{C}}_{\Omega}^{\nu}(\ell), 0\right): \ell>0\right\}$ and its solutions $\left\{\psi_{j}^{\ell}\right\}$. Under the same convergence assumption as above, the limiting function $\psi$ solves the equation

$$
-\Delta \psi(x)+V(x) \psi(x)=\nu k^{2} \psi(x)
$$

with Dirichlet condition, $\psi(x)=0$ for $x \in \partial \Omega$.

Let us stress an important feature, namely that the described result has a local character. It is especially important from the viewpoint of the example discussed below, where we will violate regularity of the solution at a fixed points by attaching leads to $\Omega$. This means that the solution has a singularity at such a junction, a logarithmic one for $\nu=2$, which enters the coupling between the billiard and the lead. Outside the connection points, however, the graph approximants do still converge to solution of the appropriate Schrödinger equation. 


\section{Example: Sinai billiard graphs}

We will consider a rectangular $N \times N$ lattice graph with a circular part removed reminiscent of a Sinai billiard which according to the above result such a structure can approximate, cf. Fig. 1. For practical calculations we choose $N=97$ and $\alpha_{j}=0, U_{j n}=0$; at the graph boundary we impose Dirichlet conditions. The lattice graph spacing is set to be $\ell=0.15$. From the numerical point of view it is more simple to evaluate a transport system since there are no problems related to the eigenvalue finding. The point is that to get the eigenvalues is numerically uneasy. We will therefore compare the transport properties in a situation when we attach a pair of leads to the graph (at the points $(14,40)$ and $(59,80)$ ) and to the billiards at the corresponding places. Adding a lead to a graph, represents no problems: the five edges at such a vertex are again coupled by Kirchhoff conditions, (2.3) with $\alpha_{j}=0$. On the other hand, coupling a billiard to leads needs an explanation.

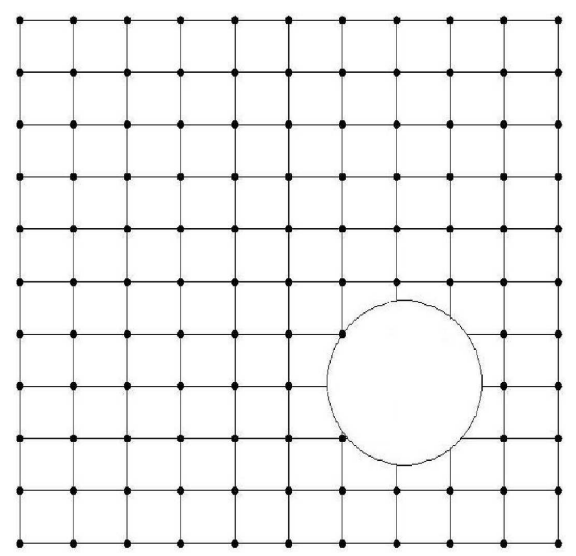

Fig. 1. Sinai billiard graph.

If we relax the regularity requirement to solution $\phi$ to a two-dimensional Helmholtz equation at a fixed point, it can have a logarithmic singularity there. It allows us to define generalized boundary values there,

$$
L_{0}(\phi):=\lim _{r \rightarrow 0} \frac{\phi(\boldsymbol{x})}{\ln r}, L_{1}(\phi):=\lim _{r \rightarrow 0}\left[\phi(\boldsymbol{x})-L_{0}(\phi) \ln r\right],
$$

where $r$ denotes the distance from the singularity. Let further $\phi_{ \pm}$be the wave functions at the leads, naturally identified with the halflines $R_{ \pm}$. They are coupled by the boundary conditions $[18]$

$$
\pm \phi_{\mp}^{\prime}\left(0_{\mp}\right)=A \phi_{\mp}\left(0_{\mp}\right)+B L_{0}(\phi), \quad L_{1}(\phi)=C \phi_{\mp}\left(0_{\mp}\right)+D L_{0}(\phi),
$$

which define a self-adjoint Hamiltonian, i.e. ensure that the probability current is conserved at the junctions, if

$A, D \in R \quad$ and $B=2 \pi \bar{C}$. 
This allows one to describe transport through the billiard and similar more complicated structures; a general scattering theory for such systems was worked out in [19].

In our particular case, suppose that the leads are attached at points $x_{1}, x_{2} \in \Omega$. The construction of the generalized eigenfunctions means to couple plane-wave solution at leads with

$$
\phi(x)=c_{1} G\left(x, x_{1} ; k\right)+c_{2} G\left(x, x_{2} ; k\right),
$$

where $G(\cdot, \cdot ; k)$ is Green's function of the Dirichlet Laplacian $-\Delta_{D}^{\Omega}$ in the billiard. The latter has a logarithmic singularity so $L_{j}(\phi)$ express in terms of $g:=G\left(x_{1}, x_{2} ; k\right)$ and

$$
\xi_{j} \equiv \xi\left(x_{j} ; k\right):=\lim _{x \rightarrow x_{j}}\left[G\left(x, x_{j} ; k\right)+\frac{\ln \left|x-x_{j}\right|}{2 \pi}\right] ;
$$

the matching conditions then determine the scattering, i.e. transmission and reflection amplitudes, as well as the coefficients $c_{1}, c_{2}$ in (4.2), similarly as in [20].

The question is, of course, what is the proper choice of the parameters in the boundary conditions (4.1). A possible way to answer it is to compare a lowenergy scattering in the system of a plane-plus-halfline to that of a similar system in which the halfline is replaced by a thin tube of radius $a$. In [21] it was shown that the two systems coincide in the leading order if we put

$$
A:=\frac{1}{2 a}, \quad D:=-\ln a, \quad B=2 \pi C=\sqrt{\frac{2 \pi}{a}} ;
$$

thus the "natural" coupling depends on a single parameter, namely radius of the "thin" component. This is chosen in the calculations to be one-tenth of the lattice graph spacing.
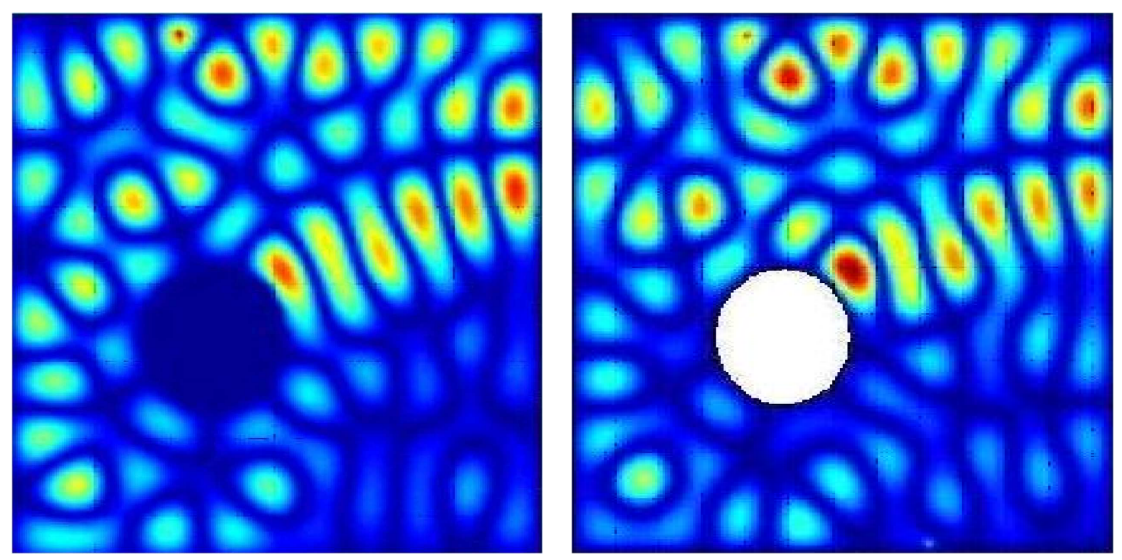

Fig. 2. Eigenfunction comparison. In the left picture the graph eigenfunction corresponds to the energy $E$ referring to the momentum $k=1.65$. The right picture shows the billiard eigenfunction of energy $2 E$. 
To demonstrate the validity of the theorems we have evaluated the solution for the graph with energy $E=(1.65)^{2}$ and compared it with a solution of the two-dimensional Sinai billiard with a doubled energy. The result is displayed in Fig. 2.

\section{Acknowledgments}

The research was supported in part by ASCR and its Grant Agency within the projects IRP AV0Z10480505 and A100480501.

\section{References}

[1] K. Ruedenberg, C.W. Scherr, J. Chem. Phys. 21, 1565 (1953).

[2] J.E. Avron, A. Raveh, B. Zur, Rev. Mod. Phys. 60, 873 (1988).

[3] P. Exner, P. Šeba, Rep. Math. Phys. 28, 7 (1989).

[4] V. Kostrykin, R. Schrader, J. Phys. A, Math. Gen. 32, 595 (1999).

[5] P. Kuchment, Waves in Random Media 12, R1 (2002).

[6] S. Albeverio, F. Gesztesy, R. Høegh-Krohn, H. Holden, Solvable Models in Quantum Mechanics, 2nd ed., AMS Chelsea, Providence (R.I.) 2005.

[7] G. Berkolaiko, E.B. Bogomolny, J.P. Keating, J. Phys. A, Math. Gen. 34, 335 (2001).

[8] T. Kottos, U. Smilansky, Phys. Rev. Lett. 79, 4794 (1997).

[9] T. Kottos, U. Smilansky, J. Phys. A, Math. Gen. 36, 3501 (2003).

[10] O. Hul, S. Bauch, P. Pakonski, N. Savytskyy, K. Życzkowski, L. Sirko, Phys. Rev. E 69, 056205 (2004).

[11] G.G. Blasdel, J. Neuroscience 12, 3139 (1992).

[12] F. Wolf, H.-U. Bauer, K. Pawelzik, T. Geisel, Nature 382, 306 (1996).

[13] G. Blum, S. Gnutzmann, U. Smilansky, Phys. Rev. Lett. 88, 114101 (2002).

[14] T. Biyikoglu, W. Hordijk, J. Leydold, T. Pisanski, P.F. Stadler, Lin. Alg. Appl. 390, 155 (2004).

[15] T. Biyikoglu, Lin. Alg. Appl. 360, 197 (2003).

[16] P. Exner, Ann. Inst. H. Poincaré: Phys. Théor. 66, 359 (1997).

[17] P. Exner, R. Gawlista, Phys. Rev. B 53, 7275 (1996).

[18] P. Exner, P. Šeba, J. Math. Phys. 28, 386 (1987).

[19] J. Brüning, V.A. Geyler, J. Math. Phys. 44, 371 (2003).

[20] P. Exner, M. Tater, D. Vaněk, J. Math. Phys. 42, 4050 (2001).

[21] P. Exner, P. Šeba, Phys. Lett. A 228, 146 (1997). 